\title{
Dimensions of Depressive Symptoms of Chinese Postpartum Mothers
}

Shwu-Ru Liou ${ }^{1}$, Qing Li $^{2}$ and Ching-Yu Cheng ${ }^{{ }^{*}}$

${ }^{1}$ College of Nursing, Chang Gung University of Science and Technology, 2, Chiapu Rd., West Sec., Putz, Chiayi, Taiwan

${ }^{2}$ College of Medicine, Medical University of South Carolina, 171 Ashley Avenue, Charleston, SC 29425, USA

\begin{abstract}
Background: The Center for Epidemiologic Studies Depression (CESD) scale, which includes both somatic and psychosocial measurements, has been widely used to measure depression; however, its use in measuring postpartum depression was limited. The purpose of the study was to test reliability and validity of the Chinese CESD when used on Chinese postpartum mothers. Dimension of depressive symptoms of Chinese postpartum mothers was analyzed as well.

Methods: The study used a cross-sectional correlational design and included a pilot and a main study. Thirty Chinese postpartum mothers participated in the pilot study, which tested the readability and content validity of the Chinese CESD. Data from 120 Chinese postpartum mothers living in the United States were analyzed in the main study. Most mothers were homemakers, highly educated, primiparous, and gave birth vaginally. Instruments used included the Chinese CESD, mentally unhealthy days, and postpartum feelings. Cronbach's alpha, Pearson correlation, and confirmatory factor analysis were applied to test the reliability and validity of the Chinese CESD.

Results: Cronbach's alpha for the Chinese CESD was .89. The convergent and divergent validities of the Chinese CESD were supported. Confirmatory factor analysis showed a three-factor model (Somatic Symptoms/Depressed Affect, Positive Affect, and Interpersonal Problems/Depressed) fit better with the data. The three factors correlated with each other ( $\mathrm{r}$ ranged from .59 to .71 ).

Conclusion: The Chinese CESD was a reliable and valid instrument for measuring depressive symptoms in Chinese postpartum mothers. Instead of reporting depressed affect, Chinese mothers tend to report physical and interpersonal problems. Healthcare professionals taking care of Chinese postpartum mothers are suggested to assess their common physical conditions as well. Future studies are recommended to include larger samples and focus on relationships between physical symptoms and depression. Comparisons of models for postpartum depression among mothers of diverse ethnicities can increase our knowledge about postpartum depression.
\end{abstract}

\section{Dimensions of Depressive Symptoms of Chinese Postpartum Mothers}

Limited researches on small samples in a few regions are not sufficient to understand the experience of postpartum depression of Chinese mothers. Lacking a clear understanding and sufficient screening for postpartum depression of Chinese mothers, as well as effective prevention and treatment, may lead to a potentially serious public health problem. A recent US national study using the Center for Epidemiologic Studies Depression Scale (CESD) on the 2001 birth cohort found that $35.6 \%$ of Chinese mothers experienced depressive symptoms at approximately nine months postpartum [1]. Of Asian mothers living in the United States who experienced moderate to severe depressive symptoms, $76.5 \%$ did not think they needed mental health care while $87.4 \%$ did not talk to healthcare professionals about their emotional or psychological problems [1]. Reasons why Chinese mothers do not seek help or report emotional problems may lie in the stigmatization of depression, perception that such feelings are a part of life, or believing it represents personal failure and loss of face [2] Not admitting they needed help became a way of coping, even though the end result was depression [3]. Since Chinese mothers tend not to report emotional problems, active screening for emotional distress becomes important.

The CESD has been widely used to measure depressive symptoms but its use in measuring postpartum depressive symptoms was limited. Different from other instruments developed or used for postpartum depression, the CESD includes dimensions of affects, somatic symptoms, and interpersonal relationships of depression. Because Chinese tend to express depression somatically rather than affectively [2], the CESD might be a better instrument to measure

\section{Publication History:}

Received: October 16, 2014

Accepted: November 24, 2014

Published: November 26, 2014

\section{Keywords:}

Chinese, Depressive symptoms, Postpartum, Reliability, Validity postpartum depressive symptoms of Chinese mothers. Therefore, this current study intended to test the reliability and validity of the Chinese CESD when used on Chinese postpartum mothers. Dimension of depressive symptoms of Chinese postpartum mothers was analyzed as well.

\section{Background}

\section{Postpartum depression among chinese mothers}

Different instruments have been applied to assess postpartum depression among Chinese mothers (including Chinese descent women in China, Taiwan, and Hong Kong). Using the Diagnostic and Statistical Manual of Mental Disorders (DSM) or Structured Clinical Interview for DSM-IV Axis I Disorders (SCID-I), the prevalence for postpartum depression was as high as $15.7 \%$ [4]. Using other screening tools (i.e. the Edinburgh Postnatal Depression Scale [EPDS], Beck Depression Inventory [BDI], CESD, and 12-item General Health Questionnaire [GHQ-12]) for postpartum depressive symptoms, the rate varied widely depending on what instrument was used and where

"Corresponding Author: Dr. Ching-Yu Cheng, College of Nursing, Chang Gung University of Science and Technology, 2, Chiapu Rd., West Sec., Putz, Chiayi, Taiwan; E-mail: chingyuus@gmail.com

Citation: Liou SR, Li Q, Cheng CY (2014) Dimensions of Depressive Symptoms of Chinese Postpartum Mothers. Int J Nurs Clin Pract 1: 104. doi: http://dx.doi. org/10.15344/2394-4978/2014/104

Copyright: @ 2014 Liou et al. This is an open-access article distributed under the terms of the Creative Commons Attribution License, which permits unrestricted use, distribution, and reproduction in any medium, provided the original author and source are credited. 
the assessment was done. For example, using the GHQ-12, the rate was $32 \%$ in Hong Kong [5]; when using the CESD, the rate was 35.3\% in Taiwan [6]; when using the BDI, the rate was $50.2 \%$ in Taiwan [7]; and when using the EPDS, the rate was $28.8 \%$ in Taiwan [8] and was $4.7 \%$ in Mainland China [9].

\section{Measurements for postpartum depression}

The EPDS, BDI, GHQ, and CESD have been developed to screen for postpartum depression and have been translated into Chinese. The EPDS is a ten-item scale, which includes psychological items but lacks of items of somatic symptoms. Its validity was initially supported with 84 mothers at six weeks postpartum [10]. The reliability of the Chinese EPDS was supported with Chinese mothers $[8,9]$. However, the EPDS may measure not only postpartum depression but also anxiety [11] The BDI and BDI-II contain 21 symptoms and attitudes specific to depression [12]. A satisfactory reliability of the Chinese BDI has been reported [7]. However, somatic items in the BDI overlap with postpartum physical symptoms [11]. The Chinese GHQ-12 showed satisfactory sensitivity and specificity in detecting postpartum depression [5]. However, confirmatory factor analysis revealed a factorial instability and variability in best fits across time [5]. The GHQ has been recommended to use for comorbid psychopathology rather than postpartum depression [11]

The CESD had been used in different samples to screen for depressive symptoms among Chinese people. However, very few studies had been done with postpartum mothers. Of the studies using the 20-item CESD that conducted factor analysis, two-, three-, four, and five-factor models were reported [13-17]. The original scale development study reported a four-factor model: depressed affect, somatic symptoms, positive affect, and interpersonal problems [13]. The two-factor model distinguished interpersonal problems from the remaining items [14]. The three-factor model loaded depressed affect partially into somatic symptoms and partially into interpersonal problems, with the remaining positive affect as the third factor [15]. A four-factor model was reported in Zhang and Norvilitis' study [16] Nine items in Zhang and Norvilitis's study cross-loaded into two or more factors. The five-factor model, depressed affect, positive effect, somatic/retarded, interpersonal, and somatic, emerged through a principal component analysis in which psychological items did not load with physical items on the same factor [17].

\section{Methods}

\section{Design and sampling}

This study used a cross-sectional design with snowball sampling. The study included a pilot study and main study. The purpose of the pilot study was to test the readability and content validity of instruments. The reliability and validity of the instrument was also pilot tested. The validity of the instrument was tested with convergent, divergent, and construct validity in the main study. Before data collection, the study protocol was approved by the Institutional Review Board of a university. Participation criteria for both the pilot and main study included women (a) who had children under one year old; (b) who identified themselves as of Chinese or Taiwanese descent; (c) who were older than 17; (d) who could read Chinese or English; and (e) whose youngest child was healthy after being born. Participants were recruited through professional referrals, distribution of paper flyers, and electronic announcements on websites. Data were collected with paper or electronic questionnaires via mail or email.

\section{Instruments}

The CESD is a 20-item, four-point (0-3) response scale. The original scale was developed for testing depressive symptomatology in the general population [13]. A score higher than 15 indicates an individual is potentially depressed. The Cronbach's alphas of the scale ranged from .84 to .90 when tested on European Americans in the community and on psychiatric patients. The $2,4,6$, and 8 weeks testretest correlations were high (ranging from .51 to .67). The convergent validity was supported by the CESD's positive correlation with scales measuring depression or psychopathology ( $\mathrm{r}$ ranged .43 to .74). The discriminant validity was evidenced by negative or low positive correlation between the CESD and scales measuring positive affects or different concepts ( $\mathrm{r}$ ranged from -.55 to .32 ). A principal component analysis revealed four factors that accounted for $40 \%$ of the total variance of depression [13].

Mentally unhealthy days: The Healthy Days Measures (HDM) is a four-item measurement that assesses an individual's perception of wellbeing [18]. The item asking mentally unhealthy days in the HDM was adopted for validity testing of the CESD. The question is "Thinking about your mental health, which includes stress, depression, and problems with emotions, for how many days during the past 30 days was your mental health not good?"

Postpartum feelings: A question that contains seven positive and seven negative feelings was adopted from a national study on postpartum mothers [19]. The seven negative feelings are discouraged, tired, isolated, messy, sad, unsure, and confused whereas the seven positive feelings are clear-headed, contented, organized, rewarded, confident, supported, and rested. Total numbers of positive and negative feelings were used to validate the CESD. The KR-20 for positive feelings was .73 and was .75 for negative feelings in the main study.

\section{Data Analysis}

The SPSS (version 13), SAS (version 9.1.3), and Mplus (version 5.0) were used to manage and analyze collected data. Cronbach's a was used to test internal consistency whereas Pearson correlation was used to evaluate test-retest reliability, convergent validity, and divergent validity. Confirmatory factor analysis using CALIS procedure in SAS first (result not shown), and then the structural equation modeling (SEM) procedures in the Mplus software package, was used to test construct validity of the CESD. The two-factor model from Cheung and Bagley's study [14], three-factor model from Gupta and Yick's study [15], and the four-factor model from Radloff's study [13] were tested because they used the 20 -item CESD and item loadings were well reported in those articles.

Analyses of the different multifactor models were conducted using weighted least squares means and variance adjusted (WLSMV) estimation method for the polychoric correlation matrix, which provided better fitting statistics in SAS than maximum likelihood method. The overall fit of the model was examined with goodnessof-fit indices, including the comparative fit index (CFI) and the root mean square error of approximation (RMSEA). Both CFI and RMSEA take into account the complexity or parsimony of the model. The CFI compares the hypothesized model and the independence model, where no relationships between the variables in the model exist. A CFI value equal to or greater than .95 indicates an excellent fit. The RMSEA compares the model to the projected population covariance matrix and assesses lack of fit. A RMSEA value lower than .05 or .06 indicates a good model fit [20]. 


\section{Results}

\section{Pilot study}

The CESD was piloted with 30 Chinese postpartum mothers. All these mothers were married and the majority of them had a high level of education. Their mean age was 33.71 ( $\mathrm{SD}=6.16)$. None of them were born in the United States and they had lived in an average of $5.38(\mathrm{SD}=3.29)$ years in the United States. Most of the mothers (63.3\%) were homemakers and $20 \%$ worked full-time. All participants indicated their most recent birth was in the United States. More than half of mothers (56.7\%) were primipara, $60 \%$ delivered vaginally, and $66.7 \%$ of infants were females. Mean infant age was $5.38(\mathrm{SD}=3.29)$ months.

For content validity, all mothers in the pilot study agreed that the translation was understandable and no comments were made on the language. The Cronbach's $\alpha$ of the CESD was .93, item-total correlation ranged from .34 to .83 , and two-week test-retest correlation was .77 . The convergent validity of the CESD was demonstrated through its positive correlation with the mentally unhealthy days $(\mathrm{r}=.83)$ and numbers of negative feelings $(\mathrm{r}=.57)$. Divergent validity was supported by the negative correlation between the CESD and numbers of positive feelings $(\mathrm{r}=-.45)$.

\section{Main study}

Data from 120 Chinese mothers who completed the CESD were analyzed. These mothers were from 18 states in the United States. The mean age of the participants was $33.15(\mathrm{SD}=5.52)$ and they had lived an average of $6.44(\mathrm{SD}=4.92)$ years in the United States. Most of them were homemakers (51.7\%) whereas $29.2 \%$ worked full-time. The majority of them (98.3\%) were born outside of the United States, $80.6 \%$ had received an educational degree of bachelor or higher, and most (73.9\%) spoke English in addition to Chinese. More than half of the participants $(62.2 \%)$ were primipara, $83(69.7 \%)$ gave birth vaginally, and $43.2 \%$ of infants were females. Mean infant age was 5.80 $(\mathrm{SD}=3.51)$ months.

In the main study, mothers experienced a mean of $7.85(\mathrm{SD}=7.34)$ days of mental unwellness in a month, $2.11(\mathrm{SD}=1.88)$ out of seven positive feelings, and $2.52(\mathrm{SD}=1.92)$ out of seven negative feelings. The mean of the CESD was 11.21 ( $\mathrm{SD}=9.23$, ranged 0 to 45$)$ and $24.5 \%$ of mothers had a score higher than 15 . The Cronbach's $\alpha$ of the CESD was .89. The convergent validity of the CESD was supported by its positive correlation with mentally unhealthy days $(r=.65)$ and the number of negative feelings $(r=.45)$. Divergent validity was supported by the negative correlation with the number of positive feelings $(\mathrm{r}=-.41)$.

Table 1 summarizes the fit of the two-, three-, and four-factor models using the WLSMV estimation method for the polychoric correlation matrix of the CESD items. The three-factor model was found to fit the observed data marginally better than any of the nested and simpler multifactor models. Although the RMSEA statistics could not achieve a good fit, the CFI statistics indicated that the three-factor model provided the best fit among these alternative models. Overall, relatively better fit was indicated for the three-factor model (Figure 1).

Using the three-factor model, participants' means score and Cronbach's $a$ for the Somatic Symptoms/Depressed Affect, Positive Affect, and Interpersonal Problems/Depressed Affect factors were $5.26(\mathrm{SD}=4.84)$ and $.83,2.66(\mathrm{SD}=3.54)$ and .78 , and $4.14(\mathrm{SD}=3.11)$ and .82 , respectively. Those three factors correlated with each other ( $\mathrm{r}$ ranged from .59 to .71). Participants who experienced depressive symptoms had significantly higher scores on factors of the Somatic Symptoms/Depressed Affect $(\mathrm{t}[43.24]=10.94, \mathrm{p}<.001, \mathrm{~d}=3.33)$, Positive Affect $(\mathrm{t}[118]=10.63, \mathrm{p}<.001, \mathrm{~d}=1.96)$, and Interpersonal Problems/Depressed Affect ( $\mathrm{t}[36.12]=7.33, \mathrm{p}<.001, \mathrm{~d}=2.44)$ than mothers who did not experience depressive symptoms.

\begin{tabular}{|c|c|c|c|c|}
\hline Item & Two-factora & $\begin{array}{l}\text { Three- } \\
\text { factorb }\end{array}$ & $\begin{array}{l}\text { Four- } \\
\text { factorc }\end{array}$ & \\
\hline V1. & Bothered by things & DA & SS/DA & SR \\
\hline V2. & Appetite poor & DA & SS/DA & SR \\
\hline V3. & $\begin{array}{l}\text { Could not shake off } \\
\text { the blues }\end{array}$ & DA & SS/DA & DA \\
\hline V4. & $\begin{array}{l}\text { As good as other } \\
\text { people }\end{array}$ & DA & PA & PA \\
\hline V5. & $\begin{array}{l}\text { Had trouble keeping } \\
\text { mind on things }\end{array}$ & DA & SS/DA & SR \\
\hline V6. & Felt depressed & DA & SS/DA & DA \\
\hline V7. & $\begin{array}{l}\text { Everything did was } \\
\text { an effort }\end{array}$ & DA & SS/DA & SR \\
\hline V8. & Hopeful about future & DA & PA & PA \\
\hline V9. & Life has been a failure & DA & $\mathrm{IP} / \mathrm{DA}$ & DA \\
\hline V10. & Felt fearful & DA & IP/DA & DA \\
\hline V11. & Restless sleep & DA & SS/DA & SR \\
\hline V12. & Felt happy & DA & PA & PA \\
\hline V13. & Talked less than usual & DA & IP/DA & SR \\
\hline V14. & Felt lonely & DA & IP/DA & DA \\
\hline V15. & $\begin{array}{l}\text { People were } \\
\text { unfriendly }\end{array}$ & IP & $\mathrm{IP} / \mathrm{DA}$ & IP \\
\hline V16. & Enjoyed life & DA & PA & PA \\
\hline V17. & Had crying spells & DA & SS/DA & DA \\
\hline V18. & Felt sad & DA & IP/DA & DA \\
\hline V19. & People dislike me & IP & IP/DA & IP \\
\hline V20. & Could not get going & DA & SS/DA & SR \\
\hline$x^{2}$ & 86.62 & 81.16 & 82.21 & \\
\hline Df & 37 & 37 & 37 & \\
\hline $\mathrm{x}^{2} / \mathrm{df}$ & 2.34 & 2.19 & 2.22 & \\
\hline CFI & .94 & .95 & .95 & \\
\hline RMSEA & .11 & .10 & .10 & \\
\hline
\end{tabular}

Table 1. Model fit for the two-, three-, and four-factor models. Note: aCheung (1998), 'bupta (2001), ${ }^{\text {cRadloff }(1977) .}$

DA: depressed affect; SS: somatic symptoms; PA: positive affect; IP: interpersonal problems; SR: somatic/retarded activity; CFI: Comparative Fit Index; RMSEA: Root Mean Square Error of Approximation

\section{Discussion}

The CESD was supported to be a reliable and valid instrument to measure depressive symptoms of Chinese postpartum mothers in this study. A three-factor model, which includes Somatic Symptoms, Positive Affect, and Interpersonal Problems, had a better fit with the data. It is interesting that Gupta and Yick's model [15], which was derived from Chinese elders, rather than Ying et al.s study [17] model derived from Chinese American students, fit this study's data from 
Citation: Liou SR, Li Q, Cheng CY (2014) Dimensions of Depressive Symptoms of Chinese Postpartum Mothers. Int J Nurs Clin Pract 1: 104. doi: http://dx.doi. org/10.15344/2394-4978/2014/104

Page 4 of 6

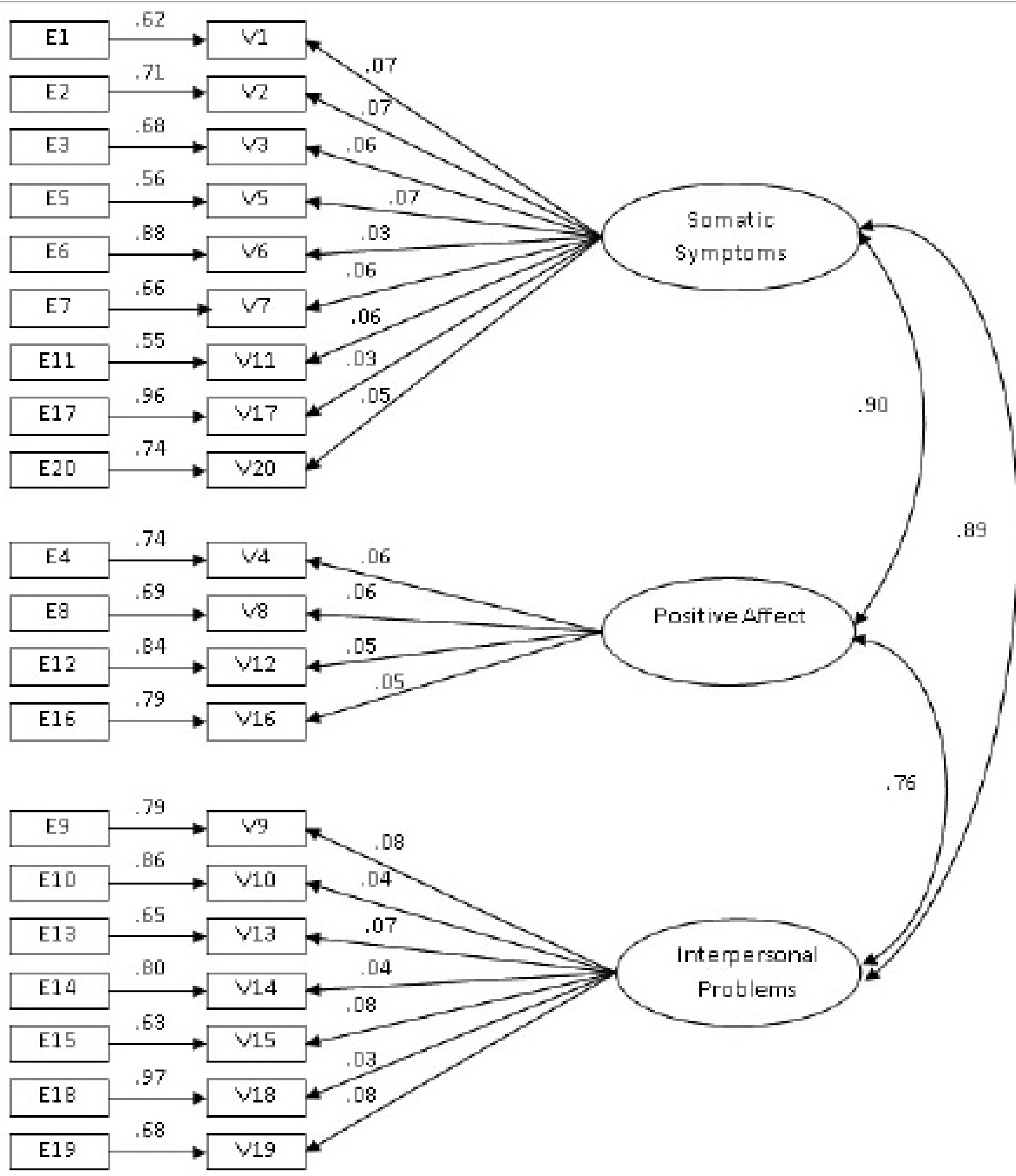

Figure 1: Revised Three-factor Model of CESD for Chinese Postpartum Mothers.

Chinese postpartum mothers better. Chinese postpartum mothers are confined to home for about a month if receiving traditional cultural care. Most participants in this study were homemakers, who might not participate in as many social activities as college students. Instead, they might have more limited social networks and spent most of their time at home taking care of their children. In addition, almost all mothers in this study were born outside of the United States, and so might not be proficient in English. Therefore, Chinese postpartum mothers' experience of depression was better explained by the model derived from elderly Chinese.
In this current study, $24.5 \%$ of Chinese mothers in the United States experienced postpartum depressive symptoms. In a national study in the United States that included Chinese mothers, 35.6\% experienced postpartum depressive symptoms [1]. Although this current study used a small sample size, the national study failed to use the Chinese version of the instrument may also be a reason of the different findings. First, providing only English instruments limited recruitment of Chinese participants who were not comfortable using English. Second, some Chinese mothers might not have fully understood scale items in English, despite claiming to know the language, and therefore 
guessed the meanings of the scale items, producing answers that might not reflect their real thoughts. Therefore, to use instruments developed in western cultures, it is necessary to translate the instruments into the language used by participants and to test their validation.

Despite the experience of acculturation, the concept and experience of depression may differ in a minority ethnic culture from that of the host culture. Chinese people have a tendency to stigmatize depression and to describe depression physically rather than psychologically [2]. As can be seen in this study's results, scale items of depressed affect in the original four-factor model were categorized into somatic symptoms and interpersonal problems in this study. In addition, mothers who experienced postpartum depressive symptoms had more somatic symptoms and interpersonal problems than mothers who did not experience postpartum depressive symptoms. Since Chinese mothers who were depressed might present physical complaints or expressed interpersonal concerns, depression also needed to be assessed and ruled out while providing care to Chinese postpartum mothers who experience physical and interpersonal problems. It is also important to consider social networks and relations while providing care to depressed mothers. Managing physical problems and improving their social skills may prevent or manage mothers' depressive symptoms.

\section{Conclusion}

The study results supported the validity of the CESD when used on Chinese postpartum mothers. In addition to depressive affect, Chinese mothers may present physical symptoms and interpersonal problems concurrently. While providing care to Chinese postpartum mothers, reports of physical conditions or social problems, which may indicate depression, need to be acknowledged. Instruments in language that mothers predominantly use need to be developed or translated to better screen for maternal health problems. The small sample size of this study limits its generalizability. Email surveys that broaden the geographic coverage may be employed in future studies. Future studies are recommended to include larger samples with various ethnicities. Comparisons of models that fit data from women of diverse ethnicities can help us understand depression and implement strategies to prevent or manage it.

\section{Author Contributions}

Dr. Liou had contributed in study design, manuscript preparation, and manuscript revision; Dr. Cheng had contributed in study design, data collection, data analysis, manuscript preparation, and manuscript revision; Dr. Li had contributed in data analysis.

\section{Acknowledgement}

The authors gratefully appreciate Dr. Lorraine O. Walker for her support and insightful guidance in conducting the study.

\section{Competing Interests}

The authors declare that they have no competing interest's exits.

\section{References}

1. Huang ZJ, Wong FY, Ronzio CR, Yu SM (2007) Depressive symptomatology and mental health help-seeking patterns of U.S.- and foreign-born mothers. Matern Child Health J 11: 257-267.

2. Chan B, Parker G (2004) Some recommendations to assess depression in Chinese people in Australasia. Aust N Z J Psychiatry 38: 141-147.
3. Chiu MYL (2004) Why Chinese women do not seek help: a cultural perspective on the psychology of women. Couns Psychol Q 17: 155-166.

4. Siu BW, Leung SS, Ip P, Hung SF, O'Hara MW (2012) Antenatal risk factors for postnatal depression: a prospective study of Chinese women at maternal and child health centres. BMC Psychiatry 12: 22

5. Ip WY, Martin CR (2006) Psychometric properties of the 12-item General Health Questionnaire (GHQ-12) in Chinese women during pregnancy and in the postnatal period. Psychol Health Med 11: 60-69.

6. Cheng CY, Walker LO, Chu TP (2013) Physical conditions and depressive symptoms of Chinese postpartum mothers in the United States and Taiwan. Health Care Women Int 34: 539-555

7. Wang SY, Jiang XY, Jan WC, Chen CH (2003) A comparative study of postnatal depression and its predictors in Taiwan and mainland China. Am J Obstet Gynecol 189: 1407-1412.

8. Kuo SY, Chen SR1, Tzeng YL2 (2014) Depression and anxiety trajectories among women who undergo an elective cesarean section. PLoS One 9: e86653.

9. Lau Y, Wang Y, Yin L, Chan KS, Guo X (2010) Validation of the Mainland Chinese version of the Edinburgh Postnatal Depression Scale in Chengdu mothers. Int J Nurs Stud 47: 1139-1151.

10. Cox JL, Holden JM, Sagovsky R (1987) Detection of postnatal depression Development of the 10-item Edinburgh Postnatal Depression Scale. $\mathrm{Br}$ Psychiatry 150: 782-786.

11. Boyd RC, Le HN, Somberg R (2005) Review of screening instruments for postpartum depression. Arch Womens Ment Health 8: 141-153.

12. Beck AT, Ward CH, Mendelson M, Mock J, Erbaugh J (1961) An inventory for measuring depression. Arch Gen Psychiatry 4: 53-63.

13. Radloff LS (1977) The CES-D Scale: A self-report depression scale for research in the general population. Appl Psychol Meas 1: 385-401.

14. Cheung CK, Bagley C (1998) Validating an American scale in Hong Kong: the Center for Epidemiological Studies Depression Scale (CES-D). J Psychol 132: 169-186.

15. Gupta R, Yick A (2001) Validation of CESD scale for older Chinese immigrants. J Ment Health Aging 7: 257-272.

16. Zhang J, Jin S (1998) Interpersonal relations and suicide ideation in China. Genet Soc Gen Psychol Monogr 124: 79-94.

17. Ying YW, Lee PA, Tsai JL, Yeh YY, Huang JS (2000) The conception of depression in Chinese American college students. Cultur Divers Ethnic Minor Psychol 6: 183-195

18. Centers for Disease Control and Prevention (2000) Measuring Healthy Days. Georgia: CDC, USA.

19. Declercq ER, Sakala C, Corry MP, Applebaum S, Risher P (2002) Listening to Mothers: Report of the First National U.S. Survey of Women's Childbearing Experiences. New York: Childbirth Connection, USA

20. Browne MW, Cudeck R (1993) Alternative ways of assessing model fit. In Bollen KA, Long JS (Ed) Testing Structural Equation Models, Sage, USA, 136-162. 